Japan. J. Math.

Vol. 12, No. 1, 1986

\title{
The non-existence of elliptic curves with everywhere good reduction over certain quadratic fields
}

\author{
By Hidenori IsHII \\ (Received December 25, 1984)
}

\section{Introduction}

The main purpose of the present paper is to show the non-existence of elliptic curves with everywhere good reduction defined over certain real quadratic fields. (See Theorem 2.3.) For this purpose we shall study $k$ rational point of order 2 of such an elliptic curve defined over a real quadratic field $k$. Then the problem is reduced to a certain Diophantine problem over $k$.

As for elliptic curves defined over imaginary quadratic field $k$, Stroeker has proved that any ellipitc curve with everywhere good reduction defined over $k$ does not have a global minimal equation by a Diophantine method in [9]. By using Stroeker's result, Setzer has shown that there exists no elliptic curve with everywhere good reduction defined over $k$ if the class number of $k$ is prime to 6 in [11]. (See also [10, p. 196].) Although the result is weaker than Setzer's, the present author has independently shown a result of the same type by a quite different method (non Diophantine method) in [2]. In $\S 3$, we shall give a mild improvement of the Theorem of [2]. (See Cor. 3.3.) In $\S 5$, we shall show some examples of elliptic curves with everywhere good reduction. In Appendix we shal give a table of elliptic curves of prime power conductor with a rational point of order 3 defined over imaginary quadratic fields with the class number one.

We should note that the problem of determining elliptic curves with everywhere good reduction defined over quadratic fields having $\boldsymbol{Q}$-rational $j$-invariant has been discussed by Setzer in [12].

$\S 1$. Let $k$ be an algebraic number field of finite degree, $\mathfrak{o}_{k}$ the maximal order of $k$ and $\mathfrak{b}_{k}^{\times}$the group of units of $k$. Any elliptic curve defined over $k$ has a plane cubic model of the form:

$$
y^{2}+a_{1} x y+a_{3} y=x^{3}+a_{2} x^{2}+a_{4} x+a_{6},
$$

where the coefficients $a_{i}$ are in $\mathfrak{b}_{k}$. Following Tate [10], we define associated 
quantities $b_{i}, c_{i}, \Delta$, and $j$ by the following formulas:

$$
\begin{aligned}
& b_{2}=a_{1}^{2}+4 a_{2}, \quad b_{4}=a_{1} a_{3}+2 a_{4}, \quad b_{6}=a_{3}^{2}+4 a_{6}, \\
& b_{8}=a_{1}^{2} a_{6}-a_{1} a_{3} a_{4}+4 a_{2} a_{6}+a_{2} a_{3}^{2}-a_{4}^{2}, \\
& c_{4}=b_{2}^{2}-24 b_{6}, \quad c_{6}=36 b_{2} b_{4}-b_{2}^{3}-216 b_{6}, \\
& \Delta=b_{4}^{3}-27 b_{6}^{2}+b_{8}\left(36 b_{4}-b_{2}^{2}\right), \quad j=c_{4}^{3} / \Delta .
\end{aligned}
$$

We call the equation (1) gobal minimal if $\operatorname{ord}_{\mathfrak{p}}(\Delta)$ is minimal for any prime ideal $\mathfrak{p}$ of $k$, where $\operatorname{ord}_{\mathfrak{p}}(\quad)$ is the additive valuation of $k$ at $\mathfrak{p}$. There exists such a global minimal equation if the class number of $k$ is one. In such a case the discriminant $\Delta$ of a global minimal equation of an elliptic curve with everywhere good reduction defined over $k$ is a unit of $k$.

§. Let $k$ be a real quadratic field and $\sigma$ be a generator of $\mathrm{Gal}(k / \boldsymbol{Q})$. We consider the following Diophantine equation:

$$
2^{6} \varepsilon_{1}+\varepsilon_{2}=a^{2},
$$

where $\varepsilon_{1}$ and $\varepsilon_{2}$ are units of $k$ and $a$ is an integer of $k$. Then we can prove the following.

LemMa 2.1. Lel $m$ be the discriminant of $k$ and suppose that $m \equiv 1$ $\bmod 4$. Let $u_{0}\left(u_{0}>1\right)$ be a fundamental unit and suppose that $N_{k / Q}\left(u_{0}\right)=-1$.

(i) If $m \neq 41,65$, the equation (2) has no solution.

(ii) If $m=41$, the soultions of (2) are given by $\left(\varepsilon_{1}, \varepsilon_{2}, a\right)=\left(\varepsilon^{2} u_{0}, \varepsilon^{2}, \varepsilon u_{0}\right)$, $\left(\varepsilon^{2} u_{0}^{\sigma}, \varepsilon^{2}, \varepsilon u_{0}^{\sigma}\right)$, where $\varepsilon$ is any unit of $k$.

(iii) If $m=65$, the solutions of (2) are given by $\left(\varepsilon_{1}, \varepsilon_{2}, a\right)=\left(\varepsilon^{2} u_{0}^{3}, \varepsilon^{2}, \varepsilon\left(2 u_{0}^{2}-1\right)\right)$, $\left(\varepsilon^{2} u_{0}^{3 \sigma}, \varepsilon^{2}, \varepsilon\left(2 u_{0}^{2}-1\right)^{\sigma}\right),\left(\varepsilon^{2}, \varepsilon^{2}, \varepsilon \sqrt{65}\right)$, where $\varepsilon$ is any unit of $k$.

Proof. Let $\left(\varepsilon_{1}, \varepsilon_{2}, a\right)$ be a solution of (2). Then $\left(\varepsilon^{2} \varepsilon_{1}, \varepsilon^{2} \varepsilon_{2}, \varepsilon a\right)$ is also a solution for any unit $\varepsilon$. Therefore we may assume that $\varepsilon_{2}= \pm 1, \pm u_{0}$. Since $\varepsilon_{2} \equiv a^{2} \bmod 4$, we have $N_{k / Q}\left(\varepsilon_{2}\right)=1$. Therefore $\varepsilon_{2}= \pm 1$ by assumption. Furthermore it is easy to prove that $\varepsilon_{2}=1$. Put $\alpha=(a-1) / 2$. By (2), we have $\alpha(\alpha+1)$ $=2^{4} \varepsilon_{1}$.

Case (I). Assume that either $\alpha$ or $\alpha+1$ is a unit. Then we may assume that $\alpha+1$ is a unit by changing $a$ by $-a$ if necessary. Put $\alpha=2^{4} u$ and $\alpha+1$ $=v$, then $u$ and $v$ are units of $k$ and $2^{4} u+1=v$. By considering the norms of the both sides, we have $N_{k / Q}(v)=1$ and $2^{4} N_{k / Q}(u)+\operatorname{Tr}(u)=0$, where $\operatorname{Tr}(u)$ is the trace of $u$. Thus we have $u=8 \pm \sqrt{65}, a=2 u^{2}-1$ and $\varepsilon_{1}=u^{3}$.

Case (II). Assume that neither $\alpha$ nor $\alpha+1$ is a unit. In this case 2 must be decomposed into two prime factors $\mathfrak{p}$ and $\mathfrak{p}^{\sigma}$ in $k$, and $\alpha \mathfrak{v}_{k}=\mathfrak{p}^{4},(\alpha+1) \mathfrak{D}_{k}=\mathfrak{p}^{\sigma 4}$. Put $\alpha+1=u \alpha^{\sigma}$, where $u$ is a unit of $k$. Then $N_{k / Q}(\alpha)+\operatorname{Tr}(\alpha)+1=N_{k / Q}(u) N_{k / Q}(\alpha)$. 
If $N_{k / Q}(\alpha)=2^{4}$, we have $N_{k / Q}(u)=-1$ and $\operatorname{Tr}(\alpha)=-33$. Then we have $\alpha=$ $(-33 \pm 5 \sqrt{41}) / 2$. Hence $a=-u_{0}$ or $-u_{0}^{o}$, and we have $\varepsilon_{1}=u_{0}$ or $u_{0}^{o}$. On the other hand, if $N_{k / Q}(\alpha)=-2^{4}$, we have slutions $\left(\varepsilon_{1}, a\right)=(1, \pm \sqrt{65}),\left(u_{0}, u_{0}\right),\left(u_{0}^{o}, u_{0}^{o}\right)$ similarly. This completes the proof of Lemma 2.1.

Let $p$ be a prime number such that $p \equiv 5 \bmod 8 . \quad$ Put $k=Q(\sqrt{p})$ and assume that the class number of $k$ is one. Let $E$ be an elliptic curve with everywhere good reduction defined over $k$. By the remark in $\S 1$, we can take an equation (1) of $E$ with $\Delta \in \mathfrak{o}_{k}^{\times}$. Let $P=(r, t)$ be a rational point of order 2 of $E$. Under a coordinate-transformation $x=x^{\prime}+r, y=y^{\prime}+t$, (1) is transformed to $y^{2}+a_{1}^{\prime} x y+a_{3}^{\prime} y=x^{3}+a_{2}^{\prime} x^{2}+a_{4}^{\prime} x+a_{6}^{\prime}$, where the coefficients are determined by the following;

$$
\begin{aligned}
& a_{1}^{\prime}=a_{1}, \quad a_{2}^{\prime}=a_{2}+3 r, \quad a_{3}^{\prime}=a_{3}+r a_{1}+2 t=0 . \\
& a_{4}^{\prime}=a_{4}^{2}-2 r a_{2}-t a_{1}+3 r^{2}, \quad a_{6}^{\prime}=0 .
\end{aligned}
$$

The associated $b_{i}^{\prime}$ are $b_{2}^{\prime}=b_{2}+12 r, b_{4}^{\prime}=b_{4}+r b_{2}+6 r^{2}$ and $b_{6}^{\prime}=b_{6}+2 r b_{4}+r^{2} b_{2}+4 r^{3}$ $=0$. (cf. Ogg[4], Tate [10]). Then $4 r \in \mathfrak{o}_{k}$, so $8 b_{4}^{\prime} \in \mathfrak{o}_{k}$ and $b_{2}^{\prime} \in \mathfrak{o}_{k}$. Put $b=8 b_{4}$, then we have $2^{8} \Delta=b^{2}\left(b_{2}^{\prime 2}-4 b\right)$. If $N_{k / Q}(b)$ is odd, then $b \in \mathfrak{o}_{k}^{\times}$and $b_{2}^{\prime} \equiv 0 \bmod 2$. Put $a=b_{2}^{\prime} / 2$, then we have $2^{6} \Delta=b^{2}\left(a^{2}-b\right)$. This is a contradiction by Lemma 2.1. Therefore $N_{k / Q}(b)$ is even. Since $2 \mathfrak{o}_{k}$ is a prime ideal of $k, b \equiv 0 \bmod 2$. Then we have $4 b_{4}^{\prime} \in \mathfrak{o}_{k}$ and $\operatorname{ord}_{2}(r) \geqq-1$. Hence $r \in \mathfrak{o}_{k}$ and $s \in \mathfrak{o}_{k}$. Thus we may assume that $(r, s)=(0,0)$. Then we have $a_{3}=a_{6}=0$ and $\Delta=a_{4}^{2}\left(b_{2}^{2}-2^{6} a_{4}\right)$. Since $\Delta \in \mathfrak{D}_{k}^{\times}$, we have $a_{4} \in \mathfrak{D}_{k}^{\times}$. This is a contradiction by Lemma 2.1. Thus we have;

Proposition 2.2. Let $p$ be a prime number such that $p \equiv 5 \bmod 8$, and put $k=Q(\sqrt{p})$. Let $E$ be an elliptic curve with everywhere good reduction defined over $k$. Assume that the class number of $k$ is one. Then $E$ has no rational point of order 2 .

The main theorem of this section is the following;

TheOREM 2.3. Let $p$ be a prime number such that $p \equiv 5 \bmod 8$ and $u_{0}$ be a fundamental unit of $k=Q(\sqrt{p})$. Assume the following conditions (i), (ii) and (iii).

(i) The calss number of $k$ is one.

(ii) $\operatorname{Tr}\left(u_{0}\right)$ is odd.

(iii) The class numbers of $\boldsymbol{Q}(\sqrt{-p})$ and $k\left(\sqrt{u_{0}}\right)$ are prime to $3 . \quad$ Then there exists no elliptic curve with everywhere good reduction defined over $k$.

Proof. Let $E$ be an elliptic curve with everywhere good reduction defined over $k$. Take a global minimal equation of $E$. We denote its discrim- 
inant by $\Delta$. Then $\Delta \in \mathfrak{o}_{k}^{\times}$. By the assumption (iii) the class number of $K=$ $k(\sqrt{\Delta})$ is prime to 3. Put $L=k\left({ }_{2} E\right)$, where ${ }_{2} E$ is the kernel of 2-multiplication of $E$. Then $L$ is a cyclic extension of $K$ or degree 3 by Proposition 2.2. If $E$ $\bmod 2$ is ordinary, $L$ is unramified over $K$ by [6, p. 274, Cor.]. Since the class number of $K$ is prime to 3 , this is a contradiction. Thus $E \bmod 2$ is supersingular. Than $K$ is unramified over $k$ by [6, p. 275, Prop. 12]. Since the class number of $k$ in narrow sense is one, we have $K=k$. Hence $L$ is a cyclic extension of $k$ of degree 2 which is unramified outside $\{2\}$. (cf. [8].) Since $\operatorname{Tr}\left(u_{0}\right)$ is odd, there exists no such extension of $k$. This completes the proof of Theorem 2.3.

ExAmple. Let $k=\boldsymbol{Q}(\sqrt{5})$ or $\boldsymbol{Q}(\sqrt{13})$ and $u_{0}$ a fundamental unit of $k$. Here we note that the class number of $k\left(\sqrt{u_{0}}\right)$ is one. In this case the assumptions (i), (ii) and (iii) of Theorem 2.3 are satisfied.

§3. In this section we consider elliptic curves defined over certain imaginary quadratic fields. The following lemma is easy to prove.

Lemma 3.1. Let $k$ be an imaginary quadratic field. Assume that the class number of $k$ is odd. Then there exists at most one quadratic extension of $k$ which is unramified outside $\{3\}$.

REMARK. More precisely, such an extension is $k(\sqrt{-3})$ unless $k=$ $\boldsymbol{Q}(\sqrt{-3})$. In case $k=\boldsymbol{Q}(\sqrt{-3})$, there exists no such extension.

TheORem 3.2. Let $k$ be an imaginary quadratic field. Assume that the class number of $k$ is odd. Let $E$ be a semi-stable elliptic curve defined over $k$ such that the discriminant $\Delta$ is a cube in $k$. Then, ${ }_{3} E=\mu_{3} \oplus Z / 3 Z$ as $\operatorname{Gal}(\bar{k} / k)$ modules. In particular, $E$ has a k-rational point of order 3 .

Proof. Put $L=k\left({ }_{3} E\right)$. Since $\Delta$ is a cuve, $L$ is a 2-extension of $k$ which is unramified outside $\{3\}$. In case $k=Q(\sqrt{-3})$, we have $L=k$. Hence we have ${ }_{3} E=\mu_{3} \oplus \boldsymbol{Z} / 3 \boldsymbol{Z}$ in this case. In case $k \neq \boldsymbol{Q}(\sqrt{-3})$, $\mathrm{Gal}(L / k)$ is cyclic because it has unique subgroup of index 2 by Lemma 3.1. Then a simple calculation of the class number $\bmod 3$ implies that $[L: k]=2$ or $4, \operatorname{Gal}(L / k)$ is a cyclic subgroup of 2-Sylow subgroup of $\mathrm{GL}_{2}\left(\boldsymbol{F}_{3}\right)$ which is not included in $\mathrm{SL}_{2}\left(F_{3}\right)$ because $k \neq Q(\sqrt{-3})$. It is easy to show that $\mathrm{Gal}(L / k)$ is conjugate to the subgroup generated by $\left(\begin{array}{rr}1 & 0 \\ 0 & -1\end{array}\right)$ in $\mathrm{GL}_{2}\left(F_{3}\right)$. This completes the proof.

REMARK. We should note that similar argument is discussed in [1].

Corollary 3.3. Let $k$ be an imaginary quadratic field. Assume that the 
class number of $k$ is prime to 6 . Then there exists no elliptic curve with everywhere good reduction defined over $k$.

Proof. When the class number of $k$ is one, the assertionis proved by Stroeker [9]. So we may assume that $k \neq Q(\sqrt{-3})$. Let $E$ be an elliptic curve with everywhere good reduction defined over $k$. By [2, Lemma 4], $\Delta$ is a cube in $k$. Then $E$ has a rational point of order 3 by Theorem 3.2. This contradicts [2, Lemma 3]. This completes the proof.

§4. In this section we consider rational points of order 5 of elliptic curves defined over certain real quadratic fields.

Proposition 4.1. Let $p$ be a prime number such that $p \equiv 1 \bmod 8$. Put $k=Q(\sqrt{p})$. Assume that the class number of $k$ is one. Let $E$ be an elliptic curve with everywhere good reduction defined over $k$. Then $E$ has no rational point of order 5 .

Proof. Let (1) (in $\S 1$ ) be a global minimal equation of $E$ and $P=(r, s)$ a rational point of order 5 . Since 5 is unramified in $k, r$ and $s$ are integers of $k$. Then we may assume that $P=(0,0)$. Similarly as in [3], we can transform (1) to the following;

$$
y^{2}+a b^{2} y=x^{3}+\frac{1}{4}\left(a^{2}+6 a b+b^{2}\right) x^{2}+\frac{1}{2} a b^{2}(a+b) x,
$$

where $a, b \in \mathfrak{o}_{k}$ with $a \equiv b \equiv 1 \bmod 2$. Then the discriminant $\Delta=-a^{5} b^{5}\left(a^{2}+\right.$ $\left.11 a b-b^{2}\right)$. Since $\Delta$ is a unit of $k, a, b$ and $a^{2}+11 a b-b^{2}$ are units of $k$. But it is easy to show that there exists no such pair of units. This completes the proof.

REMARK. As a byproduct of this argument, we obtain an elliptic curve 5.4 in $\S 5$.

\section{§. Examples}

In this section we consider some examples of elliptic curves with everywhere good reduction.

5.1. Let $n$ be a positive integer which is prime to 6 . Let $j$ be a root of the equation $j(j-1728)= \pm n^{12}$. In [9], Stroeker has proved that the following elliptic curve $E$ has good reduction everywhere over $k=\boldsymbol{Q}\left(\sqrt{2^{10} 3^{6} \pm n^{12}}\right)$.

$$
E: y^{2}+x y=x^{3}-\frac{36}{j-1728} x-\frac{1}{j-1728}
$$


For example in case $n=5$, the class number of $k$ is $2^{9} \cdot 3 \cdot 7$. (This is calculated by M. Kamei.)

5.2. Let $k=Q(\sqrt{733})$. The class number of $k$ is 3 .

$$
E: y^{2}+x y-u_{0} y=x^{3} ; \quad \Delta=-u_{0}^{5},
$$

where $u_{0}=(27+\sqrt{733}) / 2$. Then $E$ has good reduction everywhere over $k$.

5.3. Let $k=Q(\sqrt{7})$ and $u_{0}=8+3 \sqrt{7}$.

$$
E: y^{2}+x y=x^{3}-2 u_{0} x^{2}+u_{0}^{2} x ; \Delta=-u_{0}^{6} ; j=-15^{3} .
$$

This example is due to Stroeker [9]. It is easy to see that $\operatorname{End}(E) \otimes \boldsymbol{Q}=\boldsymbol{Q}(\sqrt{-7})$.

5.4. Ler $k=\boldsymbol{Q}(\sqrt{37})$ and $u_{0}=6+\sqrt{37}$.

$$
E: y^{2}-u_{0} y=x^{3}+a x^{2}+b x ; \quad \Delta=u_{0}^{6} ; \quad j=2^{12},
$$

where $a=\left(3 u_{0}+1\right) / 2$ and $b=\left(11 u_{0}+1\right) / 2 . \quad$ (cf. [12, P. 245].) $\quad(0,0)$ is a rational point of order 5. By [2, Cor. 2 and Lemma 2], we have $\operatorname{rank}(E)=\underline{11}(5)=0$, where 111 (5) is the 5-torsion part of the Shafarevich-Tate group of $E$. Since the strict class number of $k$ is one, we can show that $\operatorname{End}(E)=Z$ and $E$ is isomorphic to $E^{\sigma}$ over $k$ by [5], where $\sigma$ is a non-trivial automorphism of $k$.

REMARK. Recently, K. Shiota showed that this elliptic curve is isogeneous to Shimura's elliptic curve which is attached to a cusp from of $S_{2}\left(\Gamma_{0}(37)\right.$, భ). (cf. [7].)

5.5. Let $k=\boldsymbol{Q}(\sqrt{29})$ and $u_{0}=(5+\sqrt{29}) / 2$.

$$
E: y^{2}+x y+u_{0}^{2} y=x^{3} ; \quad \Delta=-u_{0}^{10} .
$$

This example is due to Tate (cf. [6]). The class number of $k(\sqrt{\Delta})$ is 3 .

The following two examples are obtained as byproducts of Lemma 2.1.

5.6. Let $k=\boldsymbol{Q}(\sqrt{41})$ and $u_{0}=32+5 \sqrt{41}$.

$$
E: y^{2}+x y=x^{3}-u_{0} x ; \quad \Delta=u_{0}^{4} .
$$

This example is due to Oort (cf. [9]). $E$ has three rational points of order 2.

5.7. Let $k=\boldsymbol{Q}(\sqrt{65})$ and $u_{0}=8+\sqrt{65}$.

$$
E: y^{2}+x y=x^{3}+8 u_{0} x^{2}+u_{0}^{3} x ; \quad \Delta=u_{0}^{6} ; \quad j=257^{3} .
$$

$E$ has also three rational points of order 2 . 


\section{APPENDIX}

Let $k=Q(\sqrt{d})$ be an imaginary quadratic field with class number 1 such that $k \neq Q(\sqrt{-3})$. We give a table of elliptic curves of prime power conductor with rational point of order 3 defined over $k$. In case $k=Q$, Miyawaki has given a table of such curves in [3]. So we are concerned only with elliptic curves which are not included in Miyawaki's table.

TABLE $\quad E: y^{2}+a_{1} x y+a_{3} y=x^{3}$.

\begin{tabular}{|l|c|c|c|}
\hline \multicolumn{1}{|c|}{$k$} & $a_{3}$ & $a_{1}$ & \multicolumn{1}{c|}{} \\
\hline $\boldsymbol{Q}(\sqrt{-1})$ & $26+9 \sqrt{-1}$ & $\sqrt{-1}+9$ & $-\sqrt{-1}(26+9 \sqrt{-1})^{3}$ \\
$\boldsymbol{Q}(\sqrt{-1})$ & $26-9 \sqrt{-1}$ & $-\sqrt{-1}+9$ & $\sqrt{-1}(26-9 \sqrt{-1})^{3}$ \\
$\boldsymbol{Q}(\sqrt{-1})$ & 1 & $\sqrt{-1}+3$ & $-9+26 \sqrt{-1}$ \\
$\boldsymbol{Q}(\sqrt{-1})$ & 1 & $-\sqrt{-1}+3$ & $-9-26 \sqrt{-1}$ \\
$\boldsymbol{Q}(\sqrt{11}$ & 1 & $3+\pi^{3}$ & $\pi^{6} \quad(\pi=(1 \pm \sqrt{-11}) / 2)$ \\
$\boldsymbol{Q}(\sqrt{-2}$ & 1 & $3+\rho^{3}$ & $-\rho^{6} \quad(\rho=1 \pm \sqrt{-2})$ \\
\hline
\end{tabular}

We should note that $N_{k / Q}(26+9 \sqrt{-1})=757$ is a prime number.

\section{References}

[1] A. Brumer and K. Kramer, The rank of elliptic curves, Duke Math. J., 44 (1977), 715-743.

[2] H. Ishii, The non-existence of elliptic curves with everywhere good reduction over certain imaginary quadratic fields, J. Math. Soc. Japan, 31 (1979), 273-279.

[3] I. Miyawaki, Elliptic curves of prime power conductor with Q-rational points of finite order, Osaka J. Math., 10 (1973), 309-323.

[ 4 ] A. P. Ogg, Abelian curves of 2-power conductor, Proc. Cambridge Philos. Soc., 62 (1966), 143-148.

[ 5 ] K. A. Ribet, Endomorphisms of semi-stable abelian varieties over number fields, Ann. of Math., 101 (1975), 555-562.

[6] J.-P. Serre, Propriétés galoisiennes des points d'ordre fini des courbes elliptiques, Invent. Math., 15 (1972), 259-331.

[7] G. Shimura, Introduction to the arithmetic theory of automorphic functions, Iwanami Shoten and Princeton University Press, 1971.

[8] G. Shimura and Y. Taniyama, Complex multiplications of abelian varieties and its applications to number theory, Publ. Math. Soc. Japan, No. 6, 1961.

[ 9 ] R. J. Stroeker, Elliptic curves defined over imaginary quadratic number fields, Thesis, University of Amsterdam, 1975.

[10] J. T. Tate, The arithmetic of elliptic curves, Invent. Math., 23 (1974), 179-206.

[11] B. Setzer, Elliptic curves over complex quadratic fields, Pacific J. Math., 74 (1978), 235-250. 
[12] B. Setzer, Elliptic curves with good reduction everywhere over quadratic fields and having rational $j$-invariant, Illinois J. Math., 25 (1981), 233-245.

Department of Mathematics

FACULTY OF SCIENCE

KYOTO UNIVERSITY 